\title{
Ein Diagramm zur Abschätzung unbekannter magnetischer Kernmomente
}

\author{
Von Oswald Riedel \\ Aus dem Max-Planck-Institut für Chemie, Mainz \\ (Z. Naturforschg. 5a, 654-657 [1951]; eingegangen am 5. Dezember 1950)
}

\begin{abstract}
Eine graphische Darstellung der magnetischen Kernmomente als Funktion der Massenzahlen mit dem Kernspin als Parameter liefert für die ug- und die gu-Kerne verhältnismäßig glatte Kurven. Die sogenannten magischen Nukleonenzahlen treten darin hervor, besonders bei niedrigen Spinwerten. Für Kerne, deren magnetisches Moment bei bekanntem Spin unbekannt ist, läßt sich aus dem Diagramm das Moment nach Vorzeichen und ungefährer Größe angeben. Die Art des Diagramms legt die Ausdehnung einer für das Deuteron bereits bekannten Vorstellung nahe: Ein Kernspin $x / 2$ soll zustandekommen durch $x$ Nukleonen. Die experimentellen $\mu$-Werte stimmen mit einem solchen Modell in bemerkenswertem Maße überein.
\end{abstract}

E ine große Zahl von magnetischen Kernmomenten sind in den letzten Jahren, namentlich durch die neuen elektrodynamischen Kernresonanz-Verfahren sehr zuverlässig und genau gemessen worden. Werte der Spins und magnetischen Kernmomente sind zusammengestellt in den Tabellen von $\mathrm{Mat}$ a $\mathrm{uch}$ und Flammersfeld ${ }^{1}$ und J. E. Mack ${ }^{2}$. Im folgenden soll gezeigt werden, daß Zusammenhänge zwischen den Spins, den magnetischen Momenten und den Massenzahlen der Kerne bestehen, welche erlauben, unbekannte magnetische Kernmomente bei bekanntem Kernspin abzuschätzen und darüber hinaus eine bestimmte Modellvorstellung nahelegen.

1. Zusammenhang zwischen magnetischen Momenten und Massenzahlen der Kerne

Der nächstliegende Versuch ist, das magnetische Kernmoment $\mu$ graphisch darzustellen als Funktion der Massenzahl A oder der Ordnungszahl Z. Diese beiden Möglichkeiten ergeben ganz ähnliche Bilder. Man erhält dabei regellos verteilte Punkte, sowohl für die ug-Kerne, d. h. die Kerne mit ungerader Protonen- und gerader Neutronenzahl, als auch für die gu-Kerne. Wenigstens ist das dann der Fall, wenn alle in den genannten Tabellen mitgeteilten $\mu$-Werte eingetragen werden. In einer inzwischen erschienenen Arbeit von Béné, Denis und Extermann ${ }^{3}$ werden dabei nicht alle gegenwärtig bekannten

$1 \mathrm{M}$ a t t a u c h- F l a m mer s f e ld, Isotopenbericht, Sonderheft der Z. Naturforschg., Tübingen 1949.

2 J. E. M a c k, Rev. mod. Physics 22, 64 [1950].

3 G. J. B éné, P. M. D en is u. R. C. Exterm a n n, Helv. Physica Acta 23, 493 [1950].
Daten berücksichtigt, weswegen jene Verfasser glauben, auf ein Hervortreten der Kerne mit „ausgezeichneten Nukleonenzahlen" (magic Numbers ${ }^{4}$ ) hinweisen zu können.

2. Zusammenhang zwischen Kernspin, magnetischem Kernmoment und Massenzahl

Ein übersichtlicheres Bild bietet dagegen ein Diagramm, in welchem der Kernspin $J$ als Parameter der Kurven $\mu=f(A)$ dient. Im Diagramm (a) der Abb. 1 sind die ug-Kerne eingetragen. Die Anfangspunkte der fünf Kurven liegen auf einer verhältnismäßig glatten Kurve S. Sie kann als „Spineinsatzkurve“ gelten, da sie die bereits bekannte Tatsache beschreibt, daß für das Vorkommen eines bestimmten Spinwertes eine jeweils andere, bestimmte Mindestmassenzahl erforderlich ist (vgl. $\left.{ }^{5}\right)$.

Während die Kurven $\mu=f(A)$ für die höheren Spins $9 / 2$ und $7 / 2$ verhältnismäßig monoton verlaufen, gibt es bei niedrigeren Spins Maxima und Minima. Ihre Lage wird durch die Neutronenzahlen $2,8,10,20,50,82$ und vielleicht auch 126 gegeben, also durch Zahlen, die der Reihe der ausgezeichneten Nukieonenzahlen angehören. Eine Ausnahme ist der Kein ${ }^{41} \mathrm{~K}$ mit $Z=19, N=22$, welcher etwas tiefer liegt als die beiden vorhergehenden Kerne mit $N=20$. Kernmomente, die über $\mu_{\mathrm{s}}$ hinausgehen, kommen nur bei der Kurve für $J=5 / 2$ vor.

Die Kurven sind so gezeichnet, daß kleinere Schwankungen unberücksichtigt bleiben, jedoch blieb auch

4 M. G. M a y e r, Physic. Rev. 74, 235 [1948].

5 O. $\mathrm{H}$ a x e l, J. H. D. Je n s en u. H. S u es s, Z. Physik 128, 295 [1950]. 
der ausnehmend niedrige $\mu$-Wert des ${ }^{153} \mathrm{Eu}(J=5 / 2)$ außer Betracht. Die instabilen, aber langlebigen ugKerne ${ }^{3} \mathrm{H},{ }^{129} \mathrm{~J},{ }^{135} \mathrm{Cs}$ und ${ }^{137} \mathrm{Cs}$ sind mit eingezeichnet. Sie treten nicht besonders hervor.

Teil (b) der Abb. 1 ist das entsprechende Diagramm für die gu-Kerne. Infolge des hier geringeren experimentellen Materials ist nur die Kurve für den Parameter $J=1 / 2$ genügend dicht mit Punkten belegt. Es tritt die magische Protonenzahl $Z=50$ hervor. Der

\begin{tabular}{|c|c|c|c|c|c|}
\hline ug-Kern & $J$ & $\mu$ & gu-Kern & $J$ & $\mu$ \\
\hline${ }^{159} \mathrm{~Tb}$ & $3 / 2$ & $+1,0$ & ${ }^{91} \mathrm{Zr}$ & $5 / 2$ & $(+1)$ \\
${ }^{165} \mathrm{Ho}$ & $7 / 2$ & $+2,4$ & ${ }^{123} \mathrm{Te}$ & $1 / 2$ & $-0,8$ \\
${ }^{169} \mathrm{Tm}$ & $1 / 2$ & $+0,8$ & ${ }^{125} \mathrm{Te}$ & $1 / 2$ & $-0,8$ \\
& & & ${ }^{183} \mathrm{~W}$ & $1 / 2$ & $+0,5$ \\
& & & ${ }^{189} \mathrm{Os}$ & $1 / 2$ & $+0,6$ \\
\hline
\end{tabular}

Tab. 1. Abgeschätzte Kernmomente (in KM).

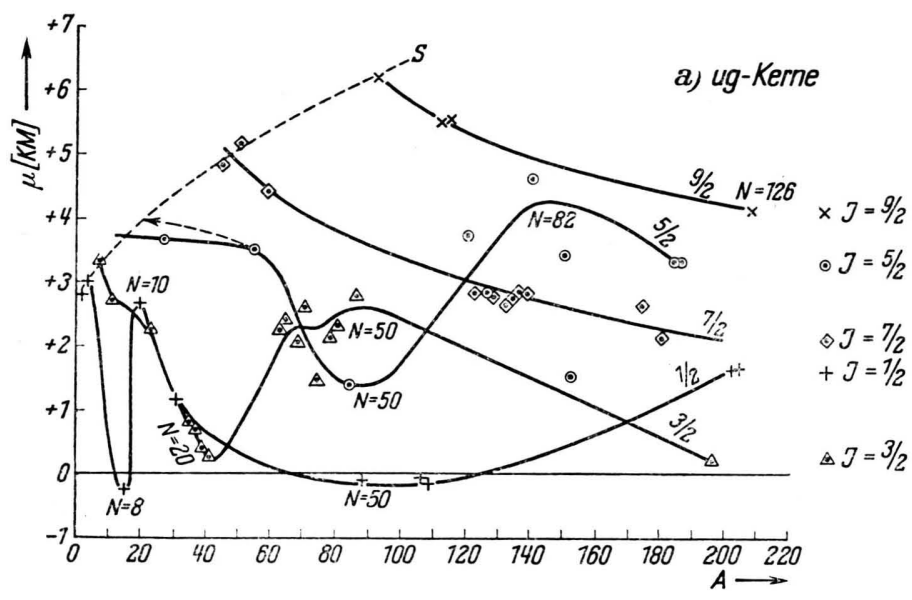

ten und den Massendefekten oder Packungsanteilen der Kerne. Beim gegenwärtigen Stand der Kernmassenbestimmung können jedoch derartige Diagramme kaum bleibende Gültigkeit besitzen, sie werden deshalb hier nicht gebracht.

\section{Abschätzungunbekannter} magnetischer Kernmomente

Der Verlauf der Kurven der ug-Kerne und der Kurve für $J=1 / 2$ der gu-Kerne kann ais im großen und ganzen gesichert betrachtet werden, da die Extrema als durch die ausgezeichneten Nukleonenzahlen bedingt zu erkennen sind. Noch unbekannte $\mu$-Werte müssen deshalb annähernd auf diesen Kurven liegen. Sind $N, Z$ und $J$ des betreffenden Kerns bekannt, so kann aus den Diagrammen ein ungefährer Wert seines Kernmoments nach Größe und Vorzeichen abgelesen werden. Für acht von zwölf Fällen dieser Art sind in Tab. 1 Werte für das Kernmoment mitgeteilt.

Leider ist nicht entscheidbar, welches

Abb. 1. Magnetisches Kernmoment $\mu$ als Funktion der Massenzahl A mit dem Kernspin $\boldsymbol{J}$ als Parameter. (a) für ug-Kerne, ungerade Protonenzahl; (b) für gu-Kerne, ungerade Neutronenzahl.

Vorzeichen das magnetische Moment des ${ }^{8} 3 \mathrm{~S}$, eines gu-Kerns mit $J=3 / 2$, hat, die Größe wurde kürzlich mit $|\mu|=0,9 \mathrm{KM}$

von den S chmidt - Diagrammen (s. z. B. ${ }^{6}$ ) her bekannte Befund zeigt sich auch hier, nämlich daß die magnetischen Kernmomente der gu-Kerne alle innerhalb des schmalen Bereiches von etwa $-2,0$ bis $+1,0$ KM liegen, während sie sich bei den ug-Kernen über einen mehr als doppelt so großen Bereich erstrecken und nur selten negativ sind. Die Spineinsatzkurve in (b) ist auf Grund einer noch darzustellenden Modellvorstellung nachträglich eingezeichnet.

Der Gedanke liegt nahe, auch einen Zusammenhang zu suchen zwischen den magnetischen Momen-

6 E. F e e n berg, Physic. Rev. 77, 771 [1950].

7 C. K. J e n, Physic. Rev. 78, 339 [1950]. bestimmt ${ }^{7}$. Nach neuen Messungen von $\mathrm{Ku} \mathrm{h}$ und W o od ga te ${ }^{8}$ hat der ug-Kern ${ }^{103} \mathrm{Rh}$ den Spin $1 / 2$ und ein wahrscheinlich negatives Moment von $0,11 \mathrm{KM}$. Da sich ein Wert $\mu=-0,11 \mathrm{KM}$ gut in das Diagramm einfügt, müßte hiernach ${ }^{103} \mathrm{Rh}$ ein negatives Moment haben.

\section{Folgerungen a us dem Diagramm}

Mit dem Spin als Parameter erhält man also sinnvolle Kurven für die $\mu$-Werte der Kerne. Diese Tatsache kann so aufgefaßt werden, daß der Spin eines

$8 \mathrm{H} . \mathrm{Kuhn}$ u. G. K. Woodgate, Nature [London] 166, 906 [1950]. 
Kerns eine Maßzahl ist für seine Struktur, wenigstens, soweit es sein magnetisches Moment betrifft. Dieser Ansicht entspricht eine schon seit längerer Zeit bekannte Modellvorstellung über den Spin und das magnetische Moment des Deuterons. Danach wird der Spin $\boldsymbol{J}=1$ des Deuterons so aufgefaßt, daß seine beiden Nukleonen, nämlich ein Proton und ein Neutron, gleichsinnig gerichtet sind. Auch sein magnetisches Moment muß deshalb die Summe der beiden Einzelmomente sein, eine Forderung, welcher der experimentelle $\mu$-Wert des Deuterons ziemlich gut entspricht, er ist etwas kleiner als erwartet. Die nächstliegende Erweiterung dieser Vorstellung auf schwerere Kerne ist die Annahme, daß ein Kernspin $J=x / 2$ zustandekommt durch $x$ gleichgerichtete Nukleonen, deren magnetische Momente sich dann ebenfalls addieren und das magnetische Moment des Kerns ergeben sollen. Die übrigen $(A-x)$ Nukleonen müssen einen abgesättigten Restkern bilden.

Der niedrigste ug-Kern ist das Proton mit $J=1 / 2$. Die höheren Kerne mit $J=1 / 2$ haben dann die Struktur $1 \mathrm{p}(\mathrm{gg})$, d. h. ein spinbestimmendes Proton und einen Restkern aus einer geraden Zahl Protonen und einer geraden Zahl Neutronen. Die höheren Spins entstehen durch Nukleonengruppen, in welchen die Zahl der Protonen immer um 1 größer ist als die Zahl der Neutronen. So hat etwa ein ug-Kern mit $J=3 / 2$ die Struktur $2 \mathrm{p} 1 \mathrm{n}(\mathrm{uu})$. Hier enthält also der Restkern außer antiparallelen pp- und nn-Paaren auch ein antiparalleles pn-Paar. Nimmt man jedoch für den Restkern eine thermische Bewegung der Nukleonen an, so wird das magnetische Moment dieses Paares im Mittel verschwinden. Entsprechende Betrachtungen gelten für die gu-Kerne, die also mit ln (gg) anfangen müssen. Die Tab. 2 enthält die zu den verschiedenen Kernspins gehörenden Strukturen und die danach berechneten Kernmomente $\mu_{\text {ber. }}$. Jeder $\mu$-Wert ist übrigens das arithmetische Mittel seiner beiden Nachbarn.

Die Kurven der Diagramme sind als Kurven gleicher Kernstruktur anzusehen. Ihre Anfangspunkte, d. h. die auf der Spineinsatzkurve S liegenden $\mu$ Werte, stimmen recht gut überein mit den berechneten Werten $\mu_{\text {ber. }}$. Diese Übereinstimmung zeigt die Tab. 3.

Von den ug-Kurven zeigt die für $J=5 / 2$ die s.chlechteste Übereinstimmung. Da ihr erster Punkt überhaupt bei einer etwas höheren Massenzahl liegt, als man aus dem sonst glatten Verlauf der S-Kurve erwartet, so sollte es hiernach noch mindestens einen Kern mit $J=5 / 2$ von geringerer Massenzahl $(A \approx 20)$

\begin{tabular}{|c|c|c|c|c|}
\hline$J$ & ug-Kern & $\mu_{\text {ber. }}$ & gu-Kern & $\mu_{\text {ber. }}$ \\
\hline $1 / 2$ & $1 p(g g)$ & $+2,79$ & $\ln (g g)$ & $-1,91$ \\
\hline $3 / 2$ & $2 p \ln (u u)$ & $+3,68$ & $\operatorname{lp} 2 \mathrm{n}(\mathrm{uu})$ & $-1,04$ \\
\hline $5 / 2$ & $3 \mathrm{p} 2 \mathrm{n}(\mathrm{gg})$ & +4 & $2 \mathrm{p} 3 \mathrm{n}(\mathrm{g} g)$ & $-0,15$ \\
\hline $7 / 2$ & $4 \mathrm{p} 3 \mathrm{n}(\mathrm{uu})$ & $+5,43$ & $3 \mathrm{p} 4 \mathrm{n}(\mathrm{uu})$ & $+0,73$ \\
\hline $9 / 2$ & $5 \mathrm{p} 4 \mathrm{n}(\mathrm{gg})$ & $+6,31$ & $4 \mathrm{p} 5 \mathrm{n}(\mathrm{gg})$ & $\begin{array}{l}+1,62 \\
\end{array}$ \\
\hline
\end{tabular}

Tab. 2. Strukturen und berechnete $\mu$-Werte (in KM).

\begin{tabular}{|c|c|c|c|c|}
\hline \multirow{2}{*}{$J$} & \multicolumn{2}{|c|}{ ug-Kerne } & \multicolumn{2}{|c|}{ gu-Kerne } \\
\hline & $\mu_{\mathrm{S}}$ & $\mu_{\text {ber. }}$ & $\mu_{\mathrm{S}}$ & $\mu_{\text {ber. }}$ \\
\hline $1 / 2$ & \multicolumn{2}{|c|}{$+2,79$} & \multicolumn{2}{|c|}{$-1,91$} \\
\hline $3 / 2$ & $+3,3$ & $+3,68$ & $-1,2$ & $-1,04$ \\
\hline $5 / 2$ & $+3,7$ & $+4,55$ & - & $-0,15$ \\
\hline $7 / 2$ & $+5,0$ & $+5,43$ & - & $+0,73$ \\
\hline $9 / 2$ & $+6,2$ & $+6,31$ & - & $+1,62$ \\
\hline
\end{tabular}

Tab. 3. Kernmomente $\mu_{\mathrm{S}}$ auf der S-Kurve und berechnete Momente $\mu_{\text {ber. }}$ in $\mathrm{KM}$.

geben. Der Pfeil in Abb. 1 (a) deutet den dann möglicherweise zu erwartenden Verlauf an.

Die Erklärung für das Zurückbleiben der gemessenen $\mu$-Werte hinter den berechneten sollte vermutlich die gleiche sein, die für das ebenfalls zu kleine magnetische Moment des Deuterons bereits herangezogen wird. Da nach neuerer Ansicht das Moment der Nukleonen durch kleinere Teilchen, etwa geladene Mesonen, erzeugt werden soll, könnte man nämlich vermuten, daß bei der Bindung mehrerer Nukleonen aneinander die momenterzeugende Bewegung dieser Mesonen behindert wird.

Für die gu-Kerne verlangt das Modell eine Variation der $\mu$-Werte in einem kleineren Bereich. Diese Forderung erfüllen die experimentellen Werte recht gut. Die S-Kurve ist hier in Näherung als Gerade eingetragen, und zwar so, daß ihre Lage ungefähr bestimmt wird durch den Anfangspunkt der Kurve für $J=3 / 2$. Als $\mu_{\mathrm{S}}$ sind eingetragen die $\mu_{\text {ber. }}$-Werte, so daß die Kurven in den durch die Pfeile angegebenen Richtungen verlaufen könnten.

Obwohl das hier dargelegte Modell etwas grundsätzlich anderes ist als ein Leuchtnukleon-Modell, wie es von $\mathrm{H}$ axe $\mathrm{l}$, J e n s e n und $\mathrm{S}$ ues s, GoeppertMayer, Nordheim oder Feenberg in verschiedenen Formen vorgeschlagen wird, stimmt es doch mit jenem in manchen Forderungen überein. Zunächst müssen, wie bereits gesagt, auch nach den Schmidt-Grenzen den ug- und den gu-Kernen verschiedene Bereiche der $\mu$-Werte zukommen. Aus der Spin-Treppenkurve bei Haxel und Mitarbb. ist ferner ebenfalls eine Spineinsatzkurve zu ersehen. Dabei 
wäre auch nach jenem Diagramm zu erwarten, daß $J=5 / 2$ eher einsetzen sollte, als bisher beobachtet.

Der Hauptunterschied besteht darin, daß hier die magnetischen Momente nicht als zufällige, zwischen den Schmidt-Grenzen liegende Werte angesehen werden, sondern daß es gerade die $\mu$-Werte sind, die die Modellvorstellung bestimmen. Diese Vorstellung scheint, wie man sieht, besonders an den Spineinsatzpunkten brauchbar zu sein. Die späteren Abweichungen treten auf an Stellen größerer Bindungsenergie, nämlich bei den magischen Nukleonenzahlen.

Im Vergleich zu den Leuchtnukleon-Modellen scheint das hier vorgetragene „Gruppen-Modell“ besonders geeignet, ein Verständnis des Verlaufs der Kurven anzubahnen, wenngleich sich noch nicht übersehen läßt, wie weit dieses Modell mit anderen Erfahrungen in Einklang gebracht werden kann.

9 Vgl. M. H a mermes h u. E. Eisner, Physic. Rev. 79, 888 [1950].

10 W. G o r dy, Physic. Rev. 76, 139 [1949].
Bemerkt sei noch, daß sich mit dem experimentell ziemlich sicher ausgeschlossenen Spinwert des Neutrons $^{9}$ von $3 / 2$ kein durch die Erfahrung gestütztes, ähnlich einfaches Modell aufbauen läßt.

\section{Quadrupolmomente}

Gordy ${ }^{10}$ zeigt an Isotopenpaaren, daß eine Beziehung besteht zwischen dem magnetischen Moment und dem Quadrupolmoment des Kerns. Für das „Gruppenmodell“ heißt das, daß auch das Quadrupolmoment $Q$ eines Kerns seiner spinbestimmenden Gruppe zu verdanken ist. Gordys Befund könnte also eine Stütze dafür sein, daß sich die Nukleonen im Restkern ungeordnet bewegen, weil in diesem Falle ein Moment irgendwelcher Art durch den Restkern nicht erzeugt werden dürfte.

Eine Kurvenschar $Q=f(A)$ mit $J$ als Parameter ist indes zur Zeit noch durch zu wenige Punkte belegt, als daß sich weitergehende Schlüsse ziehen ließen.

\title{
Über eine Methode der Variation der Anregungsbedingungen organischer Substanzen in der Glimmentladung
}

\author{
Von H. Schüler und L. Reinebeck \\ Aus der Forschungsstelle für Spektroskopie in der Max-Planck-Gesellschaft, Hechingen \\ (Z. Naturforschg. 5 a, 657-660 [1950]; eingegangen am 28. November 1950)
}

\begin{abstract}
Es wird eine Methode beschrieben, die es gestattet, die Anregungsbedingungen organischer Substanzen in der positiven Säule einer Glimmentladung zu variieren, indem man die Elektronengeschwindigkeitsverteilung in der positiven Säule durch Einführung verschiedener „Leitgase“ ändert. Die Methode gibt die Möglichkeit, die Energien, die zu der Emission bestimmter, z. Tl. neuer, Spektren notwendig sind, genauer festzulegen. Außerdem ist damit ein Weg gegeben, die zu einer Untersuchung notwendigen Substanzmengen erheblich herabzusetzen.
\end{abstract}

$\mathrm{D}$ ie Kenntnis der angeregten Elektronenzustände vielatomiger Moleküle wird durch die verschiedensten experimentellen Methoden vermittelt*.

1. Die Absorption ist die verbreitetste Methode und wird an Substanzen im gasförmigen ${ }^{1}$, flüssigen (bzw. gelösten) und festen Zustand 2 beobachtet.

2. Die Fluoreszenz ${ }^{3}$ hat den Vorteil, durch monochromatische Einstrahlung die Anregungsenergie genau festlegen zu können. Wegen ihrer Lichtschwäche wird sie aber meist

* Zusammenfassende Berichte mit Literaturangaben $1,2,3,4,6$.

${ }^{1}$ H. S p o ner u. E. T eller, Rev. modern Physics 13, 75 [1941].

2 M. M a gat u. N. M a i e r, „Spectres d'absorption des liquides, solutions et solides", Herman \& Co., Edt., Paris 1943. (Tables annuelles de constantes et données nummériques 39, Vol. XIII [1931-1936] chapitre 33.) bei höherem Dampfdruck durchgeführt, wobei Stöße 2. Art auftreten, durch die der ursprüngliche Anregungszustand des Moleküls beeinflußt wird. (Sammeln auf dem schwingungslosen Zustand des angeregten Moleküls; Auftreten von "Quenching-Prozessen“.)

3. Emission durch „heiße“ und „kalte“ Flammen 4. Diese Methode, die chemisch-thermische Prozesse benutzt, hat in letzter Zeit an Bedeutung gewonnen, da sie auch über angeregte Radikale Auskunft geben kann.

4. Die Phosphoreszenz ${ }^{3}$ gestattet durch die Beobachtung des Nachleuchtens den Nachweis von Elektronenzustän-

3 P. Pring shei m, „Fluorescence and Phosphorescence“. Interscience publishers Inc., New York-London 1949.

4 R. W. Pearse u. A. G. Gaydon, "The Identification of Molecular spectra“. Chapman \& Hall, London 1950. 